(1)

CrossMark

\title{
Early markers of cystic fibrosis structural lung disease: follow-up of the ACFBAL cohort
}

\author{
Naomi E. Wijker ${ }^{1,2,13}$, Suzanna Vidmar ${ }^{3,4,13}$, Keith Grimwood ${ }^{5,6}$, Peter D. Sly ${ }^{7}$ \\ Catherine A. Byrnes ${ }^{8}$, John B. Carlin ${ }^{3,4}$, Peter J. Cooper ${ }^{9}$, Colin F. Robertson ${ }^{10}$, \\ R. John Massie ${ }^{10}$, Mariette P.C. Kemner van de Corput ${ }^{1,2}$, Joyce Cheney ${ }^{11,12}$, \\ Harm A.W.M. Tiddens ${ }^{1,14}$ and Claire E. Wainwright (1) ${ }^{11,12,14}$ for the Australasian \\ Cystic Fibrosis Bronchoalveolar Lavage (ACFBAL) and Follow-up of the ACFBAL \\ (CF-FAB) study groups
}

@ERSpublications

In children with cystic fibrosis, airways disease severity on chest computed tomography at age $\mathbf{5}$ years increased the risk of bronchiectasis in adolescence and its extent was predicted by poorer nutrition, airway inflammation, and atelectasis http://bit.ly/2Nnk8LW

Cite this article as: Wijker NE, Vidmar S, Grimwood K, et al. Early markers of cystic fibrosis structural lung disease: follow-up of the ACFBAL cohort. Eur Respir J 2020; 55: 1901694 [https://doi.org/10.1183/ 13993003.01694-2019].

ABSTRACT Little is known about early predictors of later cystic fibrosis (CF) structural lung disease. This study examined early predictors of progressive structural lung abnormalities in children who completed the Australasian CF Bronchoalveolar Lavage (ACFBAL) clinical trial at age 5-years and participated in an observational follow-up study (CF-FAB).

Eight Australian and New Zealand CF centres participated in CF-FAB and provided follow-up chest computed-tomography (CT) scans for children who had completed the ACFBAL study with baseline scans at age 5-years. CT scans were annotated using PRAGMA-CF scoring. Ordinal regression analysis and linear regression were used to investigate associations between PRAGMA-CF (Perth-Rotterdam Annotated Grid Morphometric Analysis for CF) outcomes at follow-up and variables measured during the ACFBAL study.

99 out of 157 ACFBAL children (mean \pm SD age $13 \pm 1.5$ years) participated in the CF-FAB study. The probability of bronchiectasis at follow-up increased with airway disease severity on the baseline CT scan. In multiple regression (retaining factors at $\mathrm{p}<0.05$ ) the extent of bronchiectasis at follow-up was associated with baseline atelectasis (OR 7.2, 95\% CI 2.4-22; $\mathrm{p} \leqslant 0.001$ ), bronchoalveolar lavage (BAL) $\log _{2}$ interleukin (IL)-8 (OR 1.2, 95\% CI 1.05-1.5; p=0.010) and body mass index $\mathrm{z}$-score (OR 0.49, 95\% CI 0.24-1.00; $\mathrm{p}=0.05$ ) at age 5 years. Percentage trapped air at follow-up was associated with BAL $\log _{2}$ IL-8 (coefficient $1.3,95 \%$ CI $0.57-2.1 ; \mathrm{p}<0.001)$ at age 5 years.

The extent of airway disease, atelectasis, airway inflammation and poor nutritional status in early childhood are risk factors for progressive structural lung disease in adolescence.

This article has supplementary material available from erj.ersjournals.com

This article has an editorial commentary: https://doi.org/10.1183/13993003.00105-2020

Received: 26 Aug 2019 | Accepted after revision: 30 Dec 2019

Copyright OERS 2020 


\section{Introduction}

The lungs of patients with cystic fibrosis (CF) are thought to be structurally normal at birth. However, lower airway bacterial infection and chronic neutrophilic inflammation begin shortly afterwards $[1,2]$. Both contribute to early structural lung disease $[3,4]$ that progresses with age, resulting in variable proportions of trapped air/hypoperfusion, airway wall thickening, mucus plugging and bronchiectasis [5, 6], and a later decline in lung function [7]. Early in life the extent of bronchiectasis is generally mild, but airway wall thickening, mucus plugging and trapped air are more prominent and detected even during infancy $[3,8]$. It has been suggested that children with more severe lung disease as pre-schoolers may experience accelerated progression of structural lung disease during their school years [9], but the relationship between early life factors and later structural lung disease has not yet been well established.

The Australasian CF Bronchoalveolar Lavage (ACFBAL) study (Australian Clinical Trials Registry: ACTRN0126050006656639) was a randomised controlled trial conducted during 1999-2009. The ACFBAL study was designed to examine the clinical value of using bronchoalveolar lavage (BAL) to diagnose lower airway infection in infants and pre-school children with CF [10]. Subjects were followed for the first 5 years of life with detailed prospective collection of clinical data, including birthweight, respiratory microbiology and pulmonary exacerbations [11]. Children underwent anthropometric measurements (weight, height), a chest computed tomography (CT) scan, spirometry (forced expiratory volume in 1-s $\left.\left(\mathrm{FEV}_{1}\right)\right)$ and BAL when they completed the study at the age of 5 years. The CF-FAB study was a longitudinal observational follow-up study of the ACFBAL cohort (CF-FAB study; ACTRN12613000778785) and was conducted during 2013-2017 to assess the clinical and psychosocial changes over late childhood and adolescence, and to examine early life determinants of long-term clinical outcomes in CF.

The aim of this paper was to examine in children who participated in the ACFBAL study the association between i) clinical factors and ii) a range of measures of lung health in early childhood (up to 5 years of age) and structural lung disease in early adolescence as established by chest CT scans performed for the CF-FAB study.

\section{Methods}

\section{Study participants, setting and data sources}

Participants had completed the ACFBAL study at age 5 years and undergone a follow-up chest CT scan as part of the CF-FAB study (supplementary methods). Potential explanatory variables from the ACFBAL study included clinical and microbiological data, and age 5 years anthropometric, BAL, spirometry and CT scan outcome variables. The first chest CT scan undertaken as part of the CF FAB study between May 2013 and April 2016 when aged 9.4-15.8 years was used (figure 1). Ethics committees at each site approved the ACFBAL and CF-FAB studies and consent was obtained from parents or carer for each participant.

\section{CT scans}

ACFBAL protocols involved sequential low-dose, high-resolution CT scans without contrast. Expiratory CT slices were at three equally spaced levels. The majority of ACFBAL scans were done in children who were awake and trained to breath-hold for the scans. 18 children had scans performed under general anaesthesia for clinical or logistical reasons. CF-FAB protocols employed low-dose, spirometer-controlled inspiratory and expiratory volumetric CT scans without contrast and were all performed in children who were awake.

Affiliations: ${ }^{1}$ Pulmonology and Allergology, Erasmus Medical Center Sophia Children's Hospital, Rotterdam, The Netherlands. ${ }^{2}$ Radiology and Nuclear Medicine, Erasmus Medical Center, Rotterdam, The Netherlands. ${ }^{3}$ Clinical Epidemiology and Biostatistics Unit, Murdoch Children's Research Institute, Melbourne, Australia. ${ }^{4}$ Dept of Paediatrics, University of Melbourne, Melbourne, Australia. ${ }^{5}$ School of Medicine and Menzies Health Institute Queensland, Griffith University Gold Coast Campus, Gold Coast, Australia. ${ }^{6}$ Depts of Infectious Diseases and Paediatrics, Gold Coast Health, Gold Coast, Australia. ${ }^{7}$ Child Health Research Centre, The University of Queensland, Brisbane, Australia. ${ }^{8}$ Starship Children's Health and Dept of Paediatrics, University of Auckland, Auckland, New Zealand. 'Dept of Respiratory and Sleep Medicine, The Children's Hospital at Westmead, Sydney, Australia. ${ }^{10}$ Dept of Respiratory Medicine, Royal Children's Hospital, Melbourne, Australia.

${ }^{11}$ Dept of Respiratory and Sleep Medicine, Queensland Children's Hospital, Brisbane, Australia. ${ }^{12}$ School of Medicine, The University of Queensland, Brisbane, Australia. ${ }^{13} \mathrm{~N}$.E. Wijker and S. Vidmar contributed equally to this paper. ${ }^{14}$ H.A.W.M. Tiddens and C.E. Wainwright contributed equally to this paper.

Correspondence: Harm A.W.M. Tiddens, Wytemaweg 80, 3015 CN Rotterdam, The Netherlands. E-mail: h. tiddensđerasmusmc.nl 
FIGURE 1 Timeline for collection of data and relationship between the ACFBAL and CF-FAB studies. ACFBAL: Australasian Cystic Fibrosis Bronchoalveolar Lavage study; BAL: bronchoalveolar lavage; CF-FAB: Follow-up of the Australasian Cystic Fibrosis Bronchoalveolar Lavage study; CT: computed tomography.

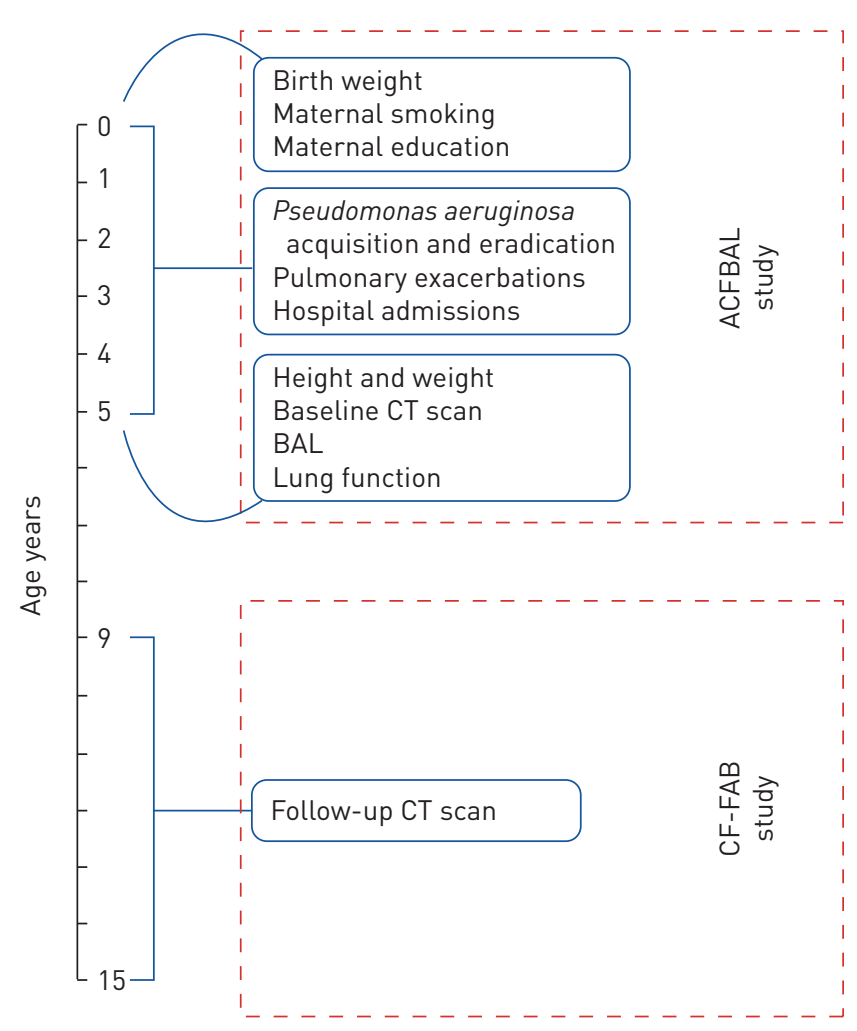

\section{Image analysis}

As different CT scan imaging techniques were used for the ACFBAL and CF-FAB studies, CT scan outcome variables were not directly comparable by visual inspection. We analysed the CT scans using the Perth-Rotterdam Annotated Grid Morphometric Analysis for CF (PRAGMA-CF) method. Briefly, with PRAGMA-CF [12] for inspiratory CT scans, a grid overlies 10 equally spaced axial slices. Grid cells were then annotated in hierarchical order: i) bronchiectasis; ii) mucus plugging; iii) airway wall thickening; iv) atelectasis; and v) normal. For expiratory scans, grid cells were annotated as trapped air, normal or as atelectasis. For each sub-score, the volume fraction was expressed as percentage of total lung volume. In addition, a composite score $\left(\%_{\text {Disease }}\right)$ was computed reflecting all components related to airways disease by

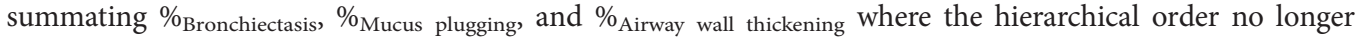
applied for this measure. ACFBAL scans were scored in random order by a single observer (observer 1) using PRAGMA-CF, while another (observer 2) scored the CF-FAB scans. Observers were blinded to clinical status and other CT scan results. 18 randomly selected ACFBAL scans were also scored by observer 2 to assess inter-observer agreement. Observer 2 also rescored 25 randomly selected CF-FAB scans 2 months after the first scoring to assess intra-observer reliability.

\section{Statistical analyses}

As different CT scan imaging techniques were employed for the ACFBAL and CF-FAB studies, CT scan outcome variables could not be analysed longitudinally using the absolute change between individual

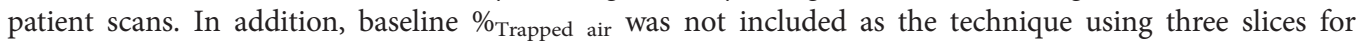
ACFBAL was less sensitive relative to the follow-up scans adopting a volumetric approach.

$\%_{\text {Bronchiectasis }}$ at follow-up was collapsed into three categories: no; minimal $(\leqslant 1 \%)$; and $>1 \%$ bronchiectasis. The $1 \%$ cut-off was the integer value closest to the median of the non-zero values. Z-scores were calculated for $\mathrm{FEV}_{1}$ [13] and nutritional measures (2000 Centers for Disease Control Growth Reference Charts: www. cdc.gov/growthcharts/cdc_charts.htm).

Regression methods examined associations between the level of bronchiectasis (ordinal regression) and

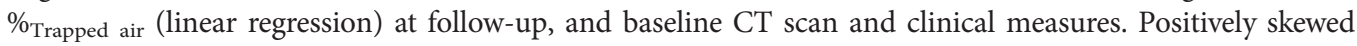
explanatory variables were transformed to logarithmic base- 2 scale, so that odds ratios and regression coefficients represent expected outcome change per doubling of the early life explanatory variable. Where extreme skewness was present, due to a large proportion of zeroes, baseline CT variables were dichotomised (bronchiectasis, atelectasis, mucus plugging) for analysis. Risk factors were examined separately adjusting for sex and age at follow-up. Those exhibiting association at p-values $<0.1$ were 
assessed in multiple regression with backward selection, retaining explanatory variables with p-values $<0.05$ in the final model. Associations are presented with $95 \%$ confidence intervals and two-sided p-values. Predicted probabilities of bronchiectasis were calculated from an ordinal regression model for each factor (where $\mathrm{p}<0.05$ ) adjusting for age at follow-up and are presented with $95 \%$ confidence intervals for no bronchiectasis and $\geqslant 1 \%$ bronchiectasis at follow-up, with age at follow-up held at its mean. As general anaesthesia may be associated with potential atelectasis seen on chest CT scans, a sensitivity analysis was undertaken excluding all the baseline scans performed under general anaesthesia to examine the association between atelectasis in the remaining baseline scans and later bronchiectasis.

Intra-class correlation coefficients (ICC) were calculated using one-way ANOVA to examine intra-observer reliability of CF-FAB scan outcome variables. Bland-Altman plots examined the inter-observer agreement for ACFBAL scan outcome variables.

Statistical analysis was performed using Stata version 15.1 (StataCorp, College Station, TX, USA).

\section{Results}

\section{Study population}

Of the 157 children who completed the ACFBAL study and were eligible to participate in the follow-up study, 99 were enrolled in CF-FAB and had a follow-up chest CT scan available at a mean \pm SD age of 13 \pm 1.5 years. In addition, of these 99 CF-FAB children, 73 had inspiratory scan data available at both baseline (at age 5 years) and follow-up time points (figure 2). Participants in the CF-FAB follow-up cohort were comparable to the original ACFBAL study cohort at the time of recruitment to ACFBAL (table 1). Follow-up characteristics of the CF FAB cohort are also summarised in table 1.

\section{Distribution of CT-scan outcome variables at baseline and follow up}

Figure 3 shows the composition of inspiratory PRAGMA-CF sub-scores at baseline and at follow-up sorted by decreasing $\%_{\text {Disease. }}$ The largest component of structural lung disease at baseline was found to be

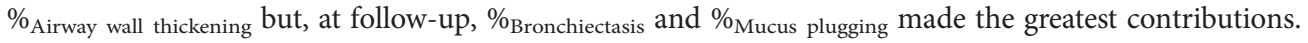

FIGURE 2 Flow chart of ACFBAL and CF-FAB CT-scans. ACFBAL: Australasian Cystic Fibrosis Bronchoalveolar Lavage study; CF: cystic fibrosis; CF-FAB: Follow-up of the Australasian Cystic Fibrosis Bron-choalveolar Lavage study; CT: computed-tomography; PRAGMACF: Perth-Rotterdam Annotated Grid Morphometric Analysis for CF.

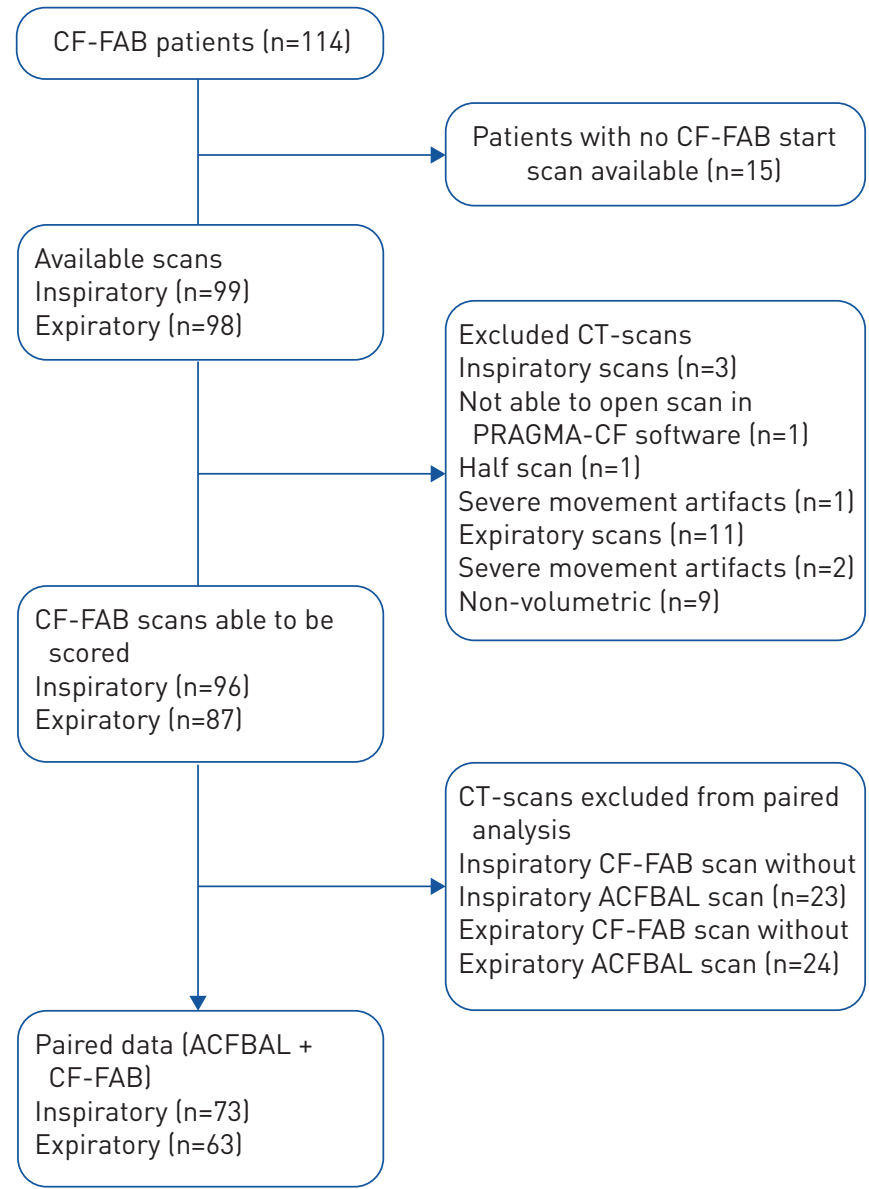


TABLE 1 Demographic and clinical characteristics comparing all the children who participated in ACFBAL and those who participated in the follow up CF-FAB study

\section{Characteristics}

At ACFBAL enrolment

Patients assessed $n$

Age months

Males

Homozygous CFTR Phe508 deletion

Pancreatic insufficient

Meconium ileus

Weight $\mathrm{kg}$

Weight z-score

Weight z-score $<-2$ SD

Sites

New Zealand

Australia

Queensland (two centres)

Victoria (two centres)

New South Wales (two centres)

South Australia

Highest education level of either parent

Patients assessed $n$

Completed year 10

Completed secondary education

Trade

Completed tertiary education

Exposure to environmental tobacco smoke

During pregnancy

Concurrent smoking in the household

Preterm delivery (<37-weeks)

Presence of respiratory symptoms

Cough

Wheeze

\section{At ACFBAL final outcome}

Patients assessed $n$

Age years

Weight z-score

BMI z-score

$\mathrm{FEV}_{1}$ z-score

Pathogenic bacterial counts in BAL $\geqslant 10^{3} \mathrm{CFU} \cdot \mathrm{mL}^{-1}$

Patients assessed $n$

None

Pseudomonas aeruginosa

Other bacterial pathogens

\section{During ACFBAL study}

Ever had Pseudomonas aeruginosa eradication

\section{At follow-up CT scan}

Age years

Weight z-score

BMI z-score

$\mathrm{FEV}_{1}$ z-score

Pathogenic bacterial counts in sputum $\geqslant 10^{3} \mathrm{CFU} \cdot \mathrm{mL}^{-1}$

None

Pseudomonas aeruginosa

Other bacterial pathogens
ACFBAL ( $n=168)$

CF-FAB (n=99) 99

168
$3.7 \pm 1.7$

$88(52)$

$111(66)$

$144(86)$

32 (19)

$5.7 \pm 1.4$

$-0.77 \pm 1.1$

23 (14)

$26(15)$

$60(36)$

40 (24)

37 (22)

$5(3.0)$

$3.6 \pm 1.6$

51 (52)

$69(70)$

84 (85)

24 (24)

$5.5 \pm 1.5$

$-0.87 \pm 1.2$

17 (17)

156

$24 \pm 15$

$39 \pm 25$

$76 \pm 49$

$17 \pm 11$

17 (17)

44 (44)

17 (17)

21 (21)

0 (0)

$35 \pm 21$

$\mathrm{n}=167 ; 53$ (32)

$\mathrm{n}=166 ; 17$ (10)

92

$12 \pm 13$

$23 \pm 25$

$45 \pm 49$

$12 \pm 13$

73 (43)

$19 \pm 19$

$\mathrm{n}=98 ; 29(30)$

$\mathrm{n}=98 ; 12$ (12)

$\mathrm{n}=166 ; 17$ (10)

$46(46)$

$\mathrm{n}=97 ; 10(10)$

157

99

$5.1 \pm 0.19$

$-0.18 \pm 0.85$

$5.1 \pm 0.13$

$0.02 \pm 0.88$

$-0.19 \pm 0.75$

$-0.02 \pm 0.76$

$\mathrm{n}=127 ;-0.58 \pm 1.3$

$\mathrm{n}=81 ;-0.59 \pm 1.3$

151

95

49 (32)

16 (11)

27 (28)

86 (57)

$15(16)$

$53(56)$

83 (53)

56 (57)

( $\mathrm{n}=99$ )

N/A

$13 \pm 1.5$

$\mathrm{N} / \mathrm{A}$

$-0.32 \pm 0.71$

N/A

$-0.20 \pm 0.68$

N/A

$-1.4 \pm 1.4$

63 (64)

9 (9.1)

27 (27)

Data are presented as mean \pm SD or $n(\%)$, unless otherwise stated. ACFBAL: Australasian Cystic Fibrosis Bronchoalveolar Lavage; BAL study: bronchoalveolar lavage; BMI: body-mass index; CF-FAB: Cystic Fibrosis-Follow-up of the ACFBAL study; CFTR: cystic fibrosis transmembrane conductance regulator; CFU: colony-forming units; CT: computed tomography; FEV ${ }_{1}$ : forced expiratory volume in $1 \mathrm{~s} ; \mathrm{N} / \mathrm{A}$ : not applicable. 

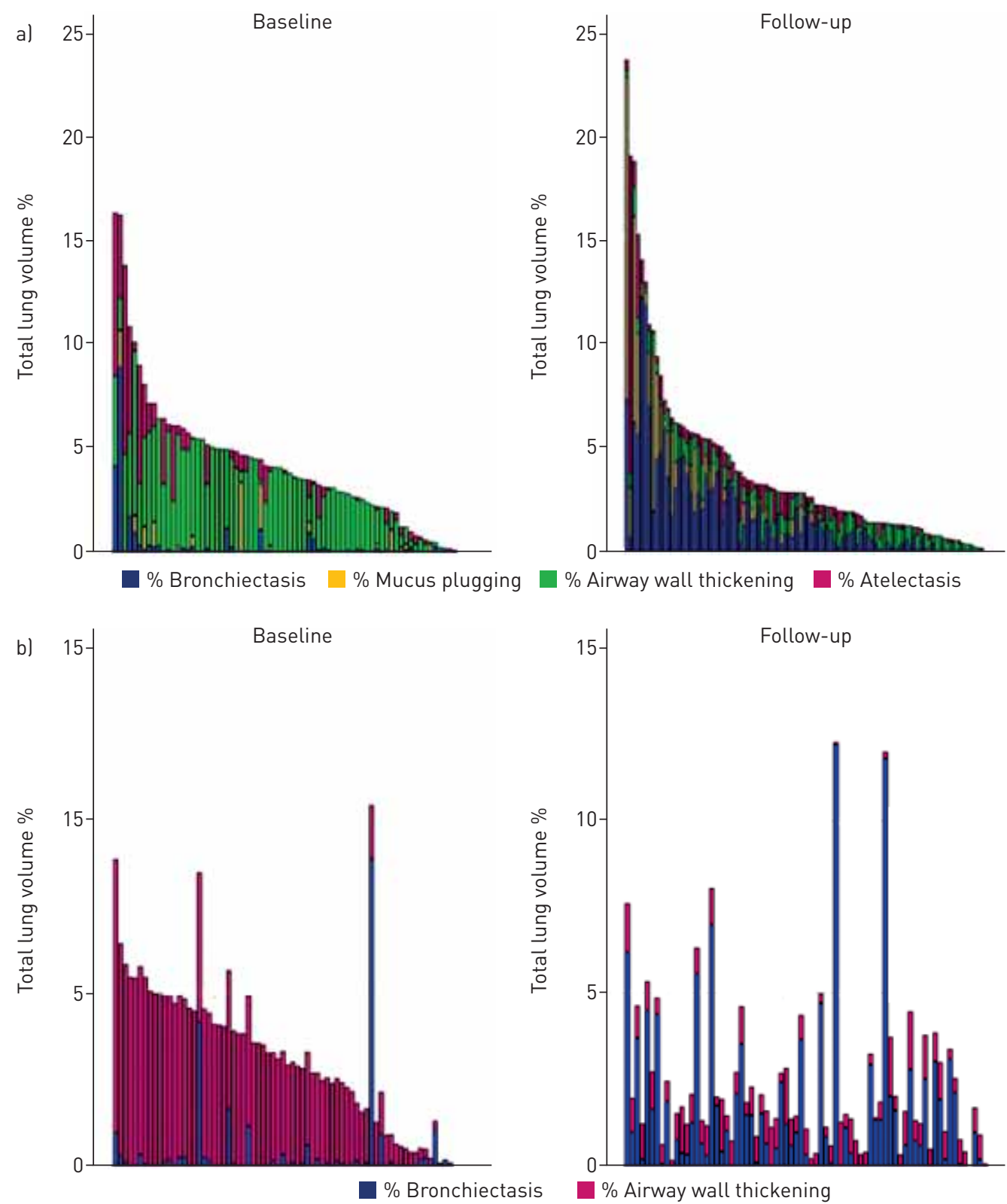

FIGURE 3 a) Overall distribution of computed-tomography scan outcome variables at baseline and at follow-up for inspiratory outcome variables. Each sub-score is expressed as a \% of the total annotated lung volume. Subjects are sorted based on the sum of \%Bronchiectasis, \%Mucus plugging, \%Airway wall thickening and

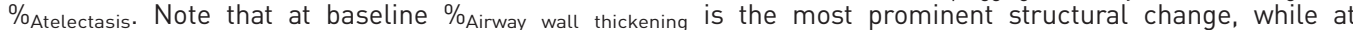
follow-up this has shifted to $\%_{\text {Bronchiectasis }}$ being more prominent. b) Distribution of $\%_{\text {Bronchiectasis }}$ and $\%_{\text {Airway }}$

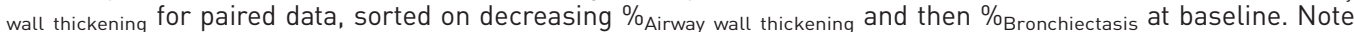
that at baseline \%Airway wall thickening is the more prominent structural change, while at follow-up this has shifted to \% ${ }_{\text {Bronchiectasis. }}$

\section{Bronchiectasis at follow-up}

Of the 96 FAB children with good quality follow-up CT scans able to be scored, 81 (84\%) had radiographic evidence of bronchiectasis, with a similar proportion (81\%) observed in the 73 children with paired baseline and follow-up CT-scans (supplementary table E1). 15 children had no radiographic signs of bronchiectasis at follow up (supplementary table E1) and the maximum lung volume affected by bronchiectasis was $12.2 \%$ with a median (interquartile range) lung volume affected by bronchiectasis of $0.88 \%(0.29-2.4)$.

Baseline atelectasis was strongly predictive of later $\%_{\text {Bronchiectasis }}$ (table 2). Presence of atelectasis was associated with a seven-fold increase in the odds of higher levels of $\%_{\text {Bronchiectasis }}$ at follow-up. In contrast, the odds of higher levels of $\%_{\text {Bronchiectasis }}$ in late childhood and adolescence were halved for every standard 


\begin{tabular}{|c|c|c|c|c|c|}
\hline \multirow[t]{2}{*}{ ACFBAL study explanatory variable } & \multicolumn{3}{|c|}{ Univariable } & \multicolumn{2}{|c|}{ Multivariable $(n=69)$} \\
\hline & $\mathbf{n}$ & OR $(95 \% \mathrm{CI})$ & p-value & OR $(95 \% \mathrm{CI})$ & p-value \\
\hline Bronchiectasis present & 73 & $2.2(0.89-5.3)$ & 0.090 & & \\
\hline Mucus plugging present & 73 & $3.8(1.2-12)$ & 0.026 & & \\
\hline \%Airway wall thickening & 73 & $1.2(0.97-1.6)$ & 0.088 & & \\
\hline Atelectasis present & 73 & $8.0(2.9-22)$ & $<0.001$ & $7.2(2.4-22)$ & $<0.001$ \\
\hline$\%_{\text {Disease }}$ & 73 & $1.3(1.07-1.7)$ & 0.012 & & \\
\hline $\begin{array}{l}\text { Pseudomonas aeruginosa ever during } \\
\text { ACFBAL }\end{array}$ & 96 & $1.6(0.73-3.5)$ & 0.248 & & \\
\hline $\begin{array}{l}\text { Pseudomonas aeruginosa eradication } \\
\text { during ACFBAL study }\end{array}$ & 96 & $1.7(0.80-3.8)$ & 0.159 & & \\
\hline $\begin{array}{l}\text { Bacteria } \\
\text { BAL }^{\#}\end{array}$ & 92 & & 0.099 & & \\
\hline None & & $\begin{array}{l}\text { Reference } \\
\text { group }\end{array}$ & & & \\
\hline Pseudomonas aeruginosa & & $3.6(0.98-13)$ & & & \\
\hline Other respiratory bacterial pathogens & & $2.3(0.90-5.9)$ & & & \\
\hline FEV $_{1}$ z-score at 5 years & 78 & $0.73(0.53-1.02)$ & 0.064 & & \\
\hline Birthweight kg & 96 & $0.72(0.35-1.5)$ & 0.366 & & \\
\hline Weight z-score at 5 years & 96 & $0.78(0.46-1.3)$ & 0.346 & & \\
\hline Height z-score at 5 years & 96 & $1.2(0.72-1.9)$ & 0.511 & & \\
\hline BMI z-score at 5 years & 96 & $0.56(0.33-0.96)$ & 0.033 & $0.49(0.24-0.999)$ & 0.050 \\
\hline $\log _{2}$ of BAL total cell count at 5 years & 88 & $0.96(0.81-1.1)$ & 0.651 & & \\
\hline $\log _{2}$ of BAL neutrophil count at 5 years & 83 & $1.01(0.90-1.1)$ & 0.898 & & \\
\hline BAL neutrophil percentage at 5 years & 86 & $1.7(0.42-6.9)$ & 0.450 & & \\
\hline $\log _{2}$ of BAL IL- 8 at 5 years & 91 & $1.2(1.08-1.4)$ & 0.002 & $1.2(1.05-1.5)$ & 0.010 \\
\hline $\begin{array}{l}\log _{2} \text { of exacerbation rate in the first } 2 \\
\text { years of life }\end{array}$ & 96 & $1.07(0.80-1.4)$ & 0.637 & & \\
\hline $\log _{2}$ of exacerbation rate during ACFBAL & 96 & $1.2(0.81-1.9)$ & 0.314 & & \\
\hline $\begin{array}{l}\log _{2} \text { of exacerbation rate resulting in } \\
\text { hospital admission during ACFBAL }\end{array}$ & 96 & $1.2(0.95-1.6)$ & 0.115 & & \\
\hline
\end{tabular}

Positively skewed explanatory variables were transformed to $\log _{2}$ scale, so that odds ratios represent expected outcome change per doubling of the early-life explanatory variable. ACFBAL: Australasian Cystic Fibrosis Bronchoalveolar Lavage study; BAL: bronchoalveolar lavage; BMI: body-mass index; CFU: colony-forming units; $\mathrm{FEV}_{1}$ : forced expiratory volume in $1 \mathrm{~s}$; IL: interleukin. \#: numbers of participants with bacterial counts $\geqslant 10^{3} \mathrm{CFU} \cdot \mathrm{mL}^{-1}$ : Pseudomonas aeruginosa $15(16 \%)$, other pathogens $52(57 \%)$, no bacterial pathogens $25(27 \%)$. ๆ: other bacterial pathogens were Staphylococcus aureus, including methicillin-resistant strains, Haemophilus influenzae, Streptococcus pneumoniae, Aspergillus spp., Stenotrophomonas maltophilia, Moraxella catarrhalis and other gram-negative bacilli. Burkholderia cepacia complex was not detected in ACFBAL participants at the age 5 years BAL [10].

deviation increase in body-mass index (BMI) at age 5 years, while there was a mean -0.18 (95\% CI $-0.33-$ -0.03; $\mathrm{p}=0.022$ ) $\mathrm{z}$-score difference in BMI between the CF FAB and ACFBAL study time points (supplementary figure E1). A doubling of the interleukin (IL)-8 concentration in BAL fluid samples at 5 years of age increased the odds of higher levels of $\%_{\text {Bronchiectasis }}$ later in life by approximately $20 \%$. No evidence of confounding was identified for maternal smoking and education at the time of recruitment to the ACFBAL study, so these covariates were not considered further. In the multiple regression model, presence of atelectasis, BMI z-score, and BAL IL-8 remained independent predictors of later bronchiectasis, with atelectasis having the strongest evidence of association (table 2). The sensitivity analysis excluding baseline scans performed under general anaesthesia confirmed that the relationship between atelectasis and bronchiectasis was not a result of general anaesthesia increasing the risk of atelectasis. Indeed, after excluding these 18 scans, the odds of higher levels of bronchiectasis at follow-up went from a seven-fold to a nine-fold increase in the presence of atelectasis (OR 9.4, 95\% CI 2.9-30; $\mathrm{p}<0.001$ ).

Using the fitted models to derive predicted probabilities illustrates the strength of the association with baseline atelectasis: the probability of bronchiectasis at $>1 \%$ was 0.77 ( $95 \%$ CI $0.58-0.89$ ) in females (at the mean age of the study group) if atelectasis was present, compared with 0.29 (95\% CI 0.14-0.49) if atelectasis was absent at baseline (with lower values but a similar difference in males). The predicted probability of remaining without bronchiectasis at follow-up was 0.25 (95\% CI 0.13-0.44) in females 
without baseline atelectasis compared with 0.04 (95\% CI $(0.01-0.11)$ in those with baseline atelectasis. Similarly, the fitted models showed the probability of bronchiectasis at $>1 \%$ was 0.77 (95\% CI $0.51-0.91$ ) in females if mucus plugging was present compared with 0.46 (95\% CI $0.30-0.63$ ) if absent at baseline, and the predicted probability of no bronchiectasis at follow-up was 0.18 (95\% CI 0.09-0.32) in females without baseline mucus plugging compared with 0.05 (95\% CI 0.02-0.17) in those with baseline mucus plugging.

The predicted probabilities and 95\% confidence intervals of follow-up bronchiectasis for each sex in the lowest (none) and highest (>1\% of lung volume) categories are shown in figure 4 for integer values of $\%_{\text {Disease }}$ BMI z-score and BAL $\log _{2}(\mathrm{IL}-8)$ levels at age 5 years. The figure demonstrates the changes in absolute risk associated with the predictor variables, providing a more direct clinical interpretation than the odds ratios reported in table 2.

Trapped air at follow-up

Neutrophil percentage and BAL IL-8 levels at age 5 years were each associated with more severe/higher $\%_{\text {Trapped air }}$ (table 3 ). In the multiple regression model, only BAL IL-8 remained after selection at $\mathrm{p}<0.1$.

\section{Reliability of scoring}

Intra-observer reliability for the scoring of CF-FAB CT-scans was generally excellent with ICCs $\geqslant 0.9$ for all

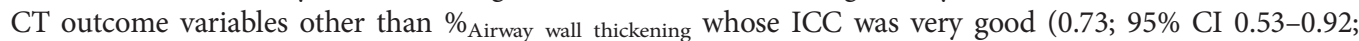

a)
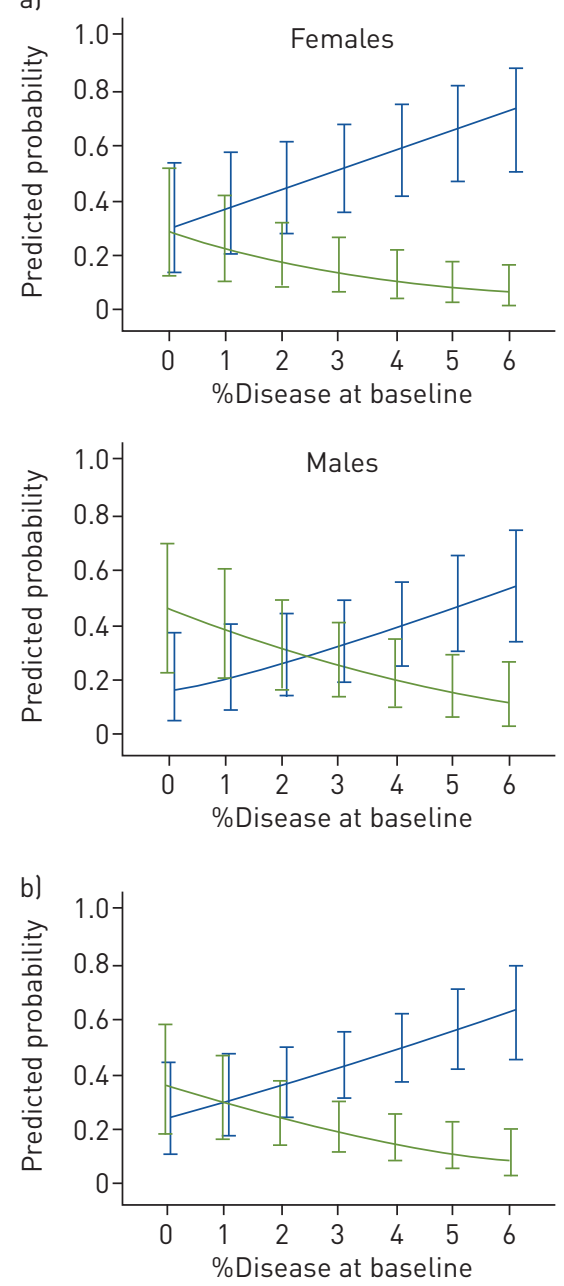
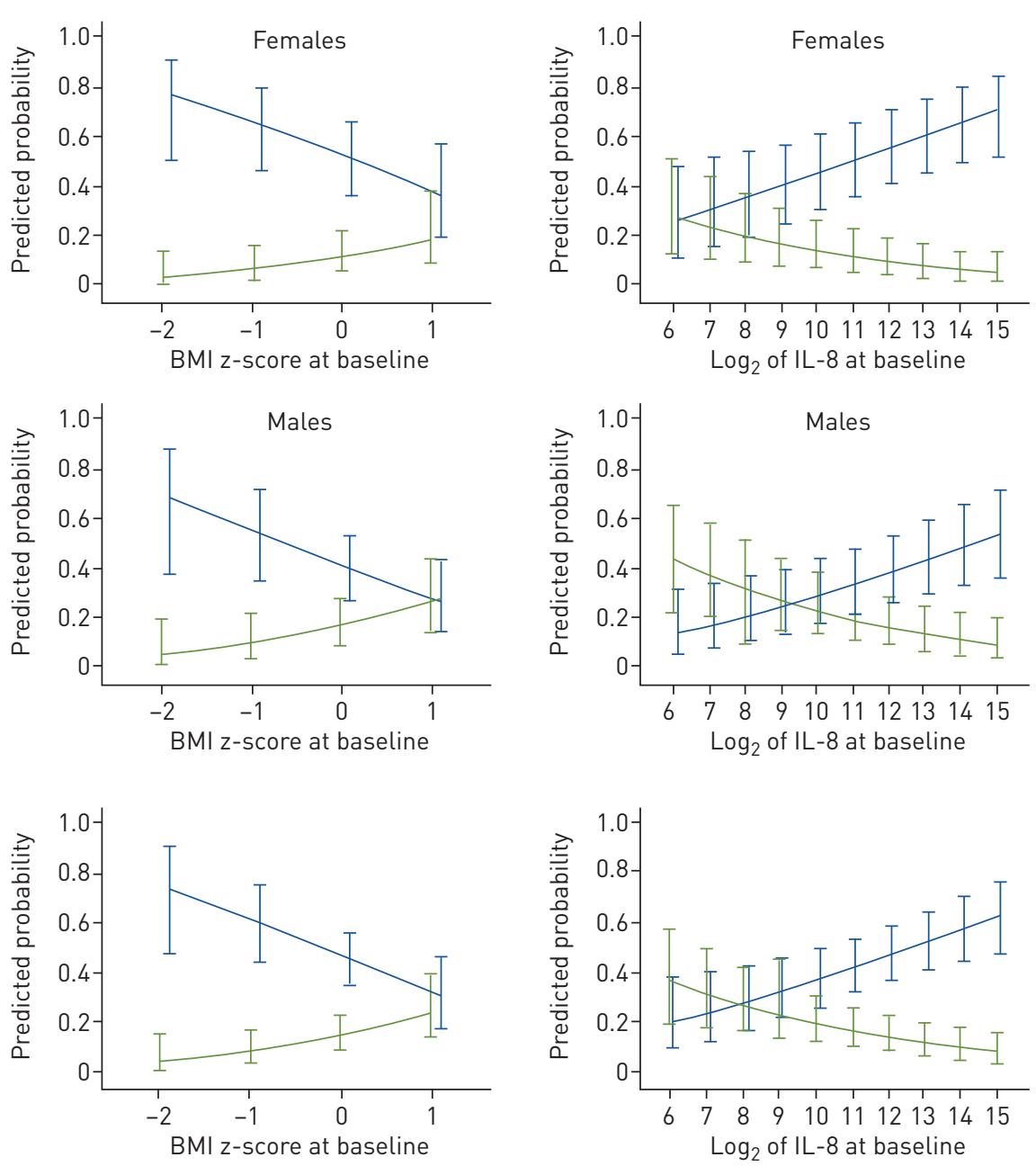

No bronchiectasis at follow-up $>1 \%$ bronchiectasis at follow-up

FIGURE 4 Predicted probabilities $(95 \% \mathrm{Cl}$ ) of no bronchiectasis and $>1 \%$ bronchiectasis at follow-up for integer values of $\%$ Disease, body mass

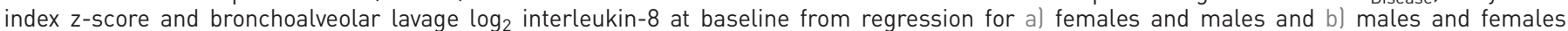

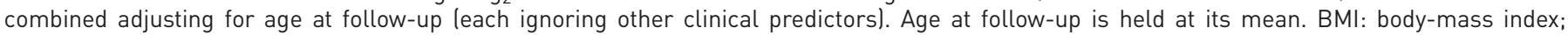
IL: interleukin. 
TABLE 3 Results from linear regressions for \% Trapped air at follow-up, adjusting for sex and age at follow-up

\begin{tabular}{|c|c|c|c|}
\hline \multirow[t]{2}{*}{ ACFBAL study explanatory variable } & \multicolumn{3}{|c|}{ Univariable } \\
\hline & $\mathbf{n}$ & $\begin{array}{l}\text { Coefficient } \\
(95 \% \mathrm{CI})\end{array}$ & p-value \\
\hline Bronchiectasis present & 64 & $2.9(-2.7-8.5)$ & 0.298 \\
\hline Mucus plugging present & 64 & $5.2(-1.7-12)$ & 0.136 \\
\hline \%Airway wall thickening & 64 & $-0.25(-1.8-1.3)$ & 0.744 \\
\hline Atelectasis present & 64 & $5.4(-0.11-11)$ & 0.055 \\
\hline$\%_{\text {Disease }}$ & 64 & $0.35(-0.98-1.7)$ & 0.598 \\
\hline Pseudomonas aeruginosa ever during ACFBAL & 87 & $3.8(-1.1-8.8)$ & 0.130 \\
\hline Pseudomonas aeruginosa eradication during ACFBAL & 87 & $3.3(-1.5-8.1)$ & 0.179 \\
\hline Bacteria $\geqslant 10^{3} \mathrm{CFU} \cdot \mathrm{mL}^{-1}$ in age 5 -years $\mathrm{BAL}^{\#}$ & 83 & & 0.036 \\
\hline None & & Reference group & \\
\hline Pseudomonas aeruginosa & & $7.2(-0.58-15)$ & \\
\hline Other respiratory bacterial pathogens" & & $7.2(1.6-13)$ & \\
\hline FEV $_{1}$ z-score at 5 years & 71 & $-0.87(-3.1-1.3)$ & 0.429 \\
\hline Birthweight kg & 87 & $-2.0(-6.5-2.5)$ & 0.377 \\
\hline Weight z-score at 5 years & 87 & $-1.2(-4.5-2.2)$ & 0.491 \\
\hline Height z-score at 5 years & 87 & $-1.1(-4.3-2.0)$ & 0.478 \\
\hline BMI z-score at 5 years & 87 & $-0.32(-3.7-3.0)$ & 0.848 \\
\hline $\log _{2}$ of BAL total cell count at 5 years & 79 & $0.27(-0.87-1.4)$ & 0.635 \\
\hline $\log _{2}$ of BAL neutrophil count at 5 years & 75 & $0.81(-0.08-1.7)$ & 0.075 \\
\hline BAL neutrophil percentage at 5 years & 78 & $11(1.3-20)$ & 0.026 \\
\hline $\log _{2}$ of BAL IL-8 at 5 years & 82 & $1.3(0.57-2.1)$ & 0.001 \\
\hline $\log _{2}$ of exacerbation rate in the first 2 years of life & 87 & $0.42(-1.5-2.3)$ & 0.669 \\
\hline $\log _{2}$ of exacerbation rate during ACFBAL & 87 & $-1.7(-4.5-1.1)$ & 0.232 \\
\hline $\begin{array}{l}\log _{2} \text { of exacerbation rate resulting in hospital admission during } \\
\text { ACFBAL }\end{array}$ & 87 & $0.72(-1.1-2.5)$ & 0.423 \\
\hline
\end{tabular}

Positively skewed explanatory variables were transformed to $\log _{2}$ scale, so that regression coefficients represent expected outcome change per doubling of the early-life explanatory variable. ACFBAL: Australasian Cystic Fibrosis Bronchoalveolar Lavage study; BAL: bronchoalveolar lavage; BMI: body-mass index; CFU: colony-forming units; $\mathrm{FEV}_{1}$ : forced expiratory volume in $1 \mathrm{~s}$; IL: interleukin. \#: numbers of participants with bacterial counts $>10^{3} \mathrm{CFU} \cdot \mathrm{mL}^{-1}$ : Psuedomonas aeruginosa $13(16 \%)$, other bacterial pathogens 47 (57\%), no bacterial pathogens 23 (28\%). ": other bacterial pathogens were Staphylococcus aureus, including methicillin-resistant strains, Haemophilus influenzae, Streptococcus pneumoniae, Aspergillus spp., Stenotrophomonas maltophilia, Moraxella catarrhalis and other gram-negative bacilli. Burkholderia cepacia complex was not detected in ACFBAL participants at the age 5-year BAL [10].

supplementary table E2). There was more inter-observer variability with the scoring of ACFBAL CT-scans, especially for $\%_{\text {Airway wall thickening }}$ and $\%_{\text {Trapped air }}$ (supplementary figure E2).

\section{Discussion}

In this study, we observed a change in the pattern of potentially reversible structural lung disease characterised predominantly by airway wall thickening at the age of 5 years to mucus plugging and irreversible bronchiectasis in adolescence. Atelectasis at the age of 5 years increased both the probability and the odds of higher levels of bronchiectasis in late childhood and adolescence, while the extent of

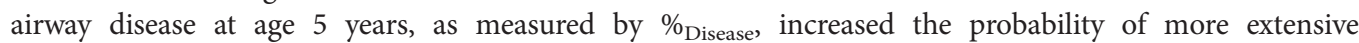
bronchiectasis in adolescence, highlighting the importance of airway disease in early life. In contrast, a larger BMI z-score at age 5 years was associated with a lower probability of ensuing bronchiectasis. These findings suggest a potential window of opportunity to intervene in early life to prevent disease progression, which should be investigated further. Airway inflammation is recognised as being associated with the development of bronchiectasis [4, 14] and in this study a doubling of IL-8 levels in BAL at age 5 years increased the odds of a higher $\%_{\text {Bronchiectasis }}$ by $20 \%$. Airway inflammation in early life was also associated with air trapping in adolescence highlighting the importance of early airway inflammation. These findings further emphasise the critical importance of establishing effective treatment in early childhood to prevent, or at least to minimise, progression of structural lung disease in children with $\mathrm{CF}$.

Atelectasis is commonly seen when there is partial or complete bronchial obstruction. The association between early atelectasis and the later development of bronchiectasis is not unexpected when one 
considers the well-recognised association between bronchiectasis and intraluminal obstruction from a foreign body [15] and with allergic bronchopulmonary aspergillosis in which bronchial obstruction, mucus plugging and atelectasis are commonly seen [16]. Animal studies have also suggested an association between bronchial obstruction and bronchiectasis, especially in the presence of airway infection and inflammation $[17,18]$.

The association between airway inflammation and later structural changes is well recognised [4, 14], however the exact pathophysiological mechanisms remain poorly understood. Similarly, while the relationship between improved nutritional status and long-term clinical outcomes, including positive effects upon survival and lung function is well established in CF [19-21], the link with bronchiectasis and air trapping and the mechanisms by which nutritional status might impact structural lung disease is unknown.

Potentially modifiable clinical factors of atelectasis, airway inflammation and poorer nutrition in early life were identified for the later development of bronchiectasis. There are, however, very few studies in young children examining the effects of improving mucus clearance on CF lung disease. It is possible that increasing airway clearance with inhaled mucoactive agents and physical therapies or by improving $\mathrm{CF}$ transmembrane conductance regulator (CFTR) function may lessen the risk of atelectasis. Modifying airway inflammation in CF is challenging because of the adverse effects associated with several anti-inflammatory agents, while also needing to maintain protective immunity in the setting of chronic airway infection. Nevertheless, airway inflammation as evidenced by elevated IL-8 levels in BAL samples at age 5 years was positively associated with both $\%_{\text {Bronchiectasis }}$ and $\%_{\text {Trapped air }}$ at follow-up, stressing the importance of early childhood airway inflammation as a potential biomarker for subsequent structural lung damage. Finally, improving nutritional status in CF is already recognised as being associated with better health outcomes and accordingly careful attention to nutrition is one of the cornerstones of early CF management.

This study did, however, have several limitations. Bronchiectasis might have been missed in the baseline CT-scans from quality differences and the protocol used, although it is unlikely that large areas of bronchiectasis would have been overlooked [5]. For the sensitive tracking of $\%_{\text {Disease }}$ over time, the same volume and CT scan protocol should be used when follow-up scans are compared to those at baseline [22]. In the current study, this was not possible and the quality of CT scan images varied significantly between baseline and follow-up for several reasons. Participating centres had different CT scanners. Also, the scanning protocol for the ACFBAL baseline CT scan differed from the one at follow-up. ACFBAL used sequential inspiratory images and three expiratory images, while at follow-up, volumetric inspiratory and expiratory scans were obtained. This could lead to underestimating the severity of structural lung abnormalities at baseline, potentially diluting the evidence for association. Another important factor in CT scan quality was that at age 5 years, children cannot co-operate as well with the breathing requirements and breath-holds as they can in later childhood [23]. The age and technical differences may have been associated with variable inflation levels and an increase in the variability of the CT scan outcome variables. Without spirometer guidance, the median lung volume of inspiratory scans in children is 77\% (range 55$106 \%$ ) of measured total lung capacity and the volume levels of expiratory scans are even more variable, with a median volume level of $140 \%$ of measured residual volume (range 83-293\%) [5]. The CT scan

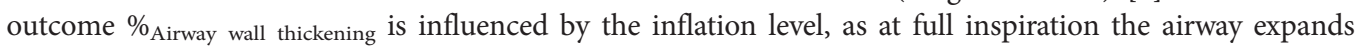
and the walls of the airway become thinner, making it more difficult to compare longitudinally [24]. However, bronchiectasis remains more clearly visible, even on end-expiratory scans and is therefore more robust as a longitudinal outcome. PRAGMA-CF has a hierarchical scoring system and therefore some caution is required when interpreting the individual components of airway disease as predictors of later bronchiectasis. However, using $\%_{\text {Disease }}$ at age 5 years takes the total components of airway disease into account and therefore avoids some of the potential concerns around differentiating between the various components of a hierarchical system. Finally, there was also a wide age range (9.4-15.8 years) for follow-up scans and so outcomes were adjusted for age [5].

Multicentre longitudinal studies involving imaging of children is challenging when comparing data over prolonged intervals, and the performance of the inspiratory and expiratory manoeuvres at different ages and CT scan quality remain important factors to be considered [22]. Automatic analysis of the scans could in the future replace manual annotations and visual scoring methods to quantify abnormal widening and thickening of the airways [25].

In conclusion, we investigated the predictive value of PRAGMA-CF at age 5 years and clinical variables over the first 5 years of life in children with CF for later school-age structural lung changes. We found that the probability of bronchiectasis in adolescence was positively related to the extent of early airway disease as measured by $\%_{\text {Disease }}$ on chest CT scans at age 5 years. Structural changes, on the chest CT scan and 
potentially modifiable clinical factors, including the BMI z-score and BAL IL-8 levels at age 5 years were independently associated with risk of bronchiectasis in adolescence. We also found that BAL IL-8 levels at 5 years of age were associated with greater levels of trapped air at follow-up. It is important to recognise that these are associations and causality cannot be inferred from this type of study. Properly conducted clinical trials however are warranted to examine whether reducing airway inflammation and atelectasis, and improving mucociliary clearance of mucus in early life, may result in less bronchiectasis in the long-term, while improving the nutritional status of children with CF may also lead to less lung damage later in life.

Acknowledgements: We would like to acknowledge Jochem Bosch for his help with the PRAGMA-CF scoring at Erasmus MC Rotterdam. We also wish to thank Nicholas Gailer at the Centre for Child Health Research Brisbane for data management support and in preparing figure 1. Finally, we wish to express our gratitude for the involvement of all the children and their families in both ACFBAL and CF-FAB studies and the research and clinical teams that supported the studies at the participating sites.

The following investigators constitute the ACFBAL Study Investigators Group: Claire E. Wainwright (Queensland Children's Hospital, Brisbane and The University of Queensland, Brisbane), Keith Grimwood (Griffith University and Gold Coast Health), Joyce Cheney (Queensland Children's Hospital, Brisbane), Narelle George (Pathology Queensland), John B. Carlin (Murdoch Children's Research Institute, Melbourne), Colin F. Robertson (Royal Children's Hospital, Melbourne), Suzanna Vidmar (Murdoch Children's Research Institute, Melbourne), Rosemary Carzino (Murdoch Children's Research Institute, Melbourne), Marj Moodie (Deakin University, Melbourne), David S. Armstrong (Monash Medical Centre, Melbourne), Peter J. Cooper (The Children's Hospital at Westmead, Sydney), A. (James) Martin (Women's \& Children's Hospital, Adelaide), Bruce Whitehead (John Hunter Children's Hospital, Newcastle), Catherine A. Byrnes (Starship Children's Hospital and The University of Auckland, Auckland), Harm A.W.M. Tiddens (Erasmus MC, Sophia Children's Hospital, Rotterdam, the Netherlands).

The following investigators constitute the CF FAB Study Investigators Group: Claire E. Wainwright (Queensland Children's Hospital, Brisbane and The University of Queensland, Brisbane), Keith Grimwood (Griffith University and Gold Coast Health), Peter D. Sly (The University of Queensland, Brisbane), Harm A.W.M. Tiddens (Erasmus MC, Sophia Children's Hospital, Rotterdam, the Netherlands), Geraint Rogers (SAHMRI and Flinders University School of Medicine, Adelaide), Richard (John) Massie (Royal Children's Hospital, Melbourne), Colin F. Robertson (Royal Children's Hospital, Melbourne), Peter J. Cooper (The Children's Hospital at Westmead, Sydney), Catherine A. Byrnes (Starship Children's Hospital and The University of Auckland, Auckland), Suzanna Vidmar (Murdoch Children's Research Institute, Melbourne), A. (James) Martin (Women's \& Children's Hospital, Adelaide), Bruce Whitehead (John Hunter Children's Hospital, Newcastle), David Armstrong (Monash Medical Centre, Melbourne), John B. Carlin (Murdoch Children's Research Institute, Melbourne), Peter Wark (John Hunter Children's Hospital and University of Newcastle, Newcastle).

Additional contributions: We are indebted to all current and former clinical and research staff from Queensland Children's Hospital, Brisbane: Nicholas Gailer, Natalie Smith, Careana Moss, Katrina Jess, Peta Yarrow; The Children's Hospital at Westmead, Sydney: Merilyn McArthur, Sam Forbes, Hiran Selvadurai; Royal Children's Hospital, Melbourne: Sarath Ranganathan, Phil Robinson, Natalie Zajakovski; Starship Children's Hospital, Auckland, Jan Tate, Rochelle Moss; Erasmus MC, Sophia Children's Hospital, Rotterdam, the Netherlands: Els Van Der Wiel.

Author contributions: Study conception and planning: C.E. Wainwright, S. Vidmar, K. Grimwood, P.D. Sly, C.A. Byrnes, J.B. Carlin, P.J. Cooper, C.F. Robertson, R.J. Massie, M.P.C. Kemner van de Corput, J. Cheney and H.A.W.M. Tiddens. Data collection: C.A. Byrnes, P.J. Cooper, C.F. Robertson, R.J. Massie, JC and C.E. Wainwright. Imaging analysis: N.E. Wijker, M.P.C. Kemner van de Corput and H.A.W.M. Tiddens. Data analysis: statistical analysis led by S. Vidmar with advice from JC. N.E. Wijker, C.E. Wainwright, H.A.W.M. Tiddens and K. Grimwood contributed to planning of statistical analysis. All authors were involved in data interpretation, and preparation, review and approval of the final manuscript.

Support statement: This study was supported by grants from the Australian National Health and Medical Research Council $(9937868,351541$ and 1044829). C.E. Wainwright was supported through Practitioner Fellowship through The Children's Hospital Foundation Brisbane (RG0692016).

Conflict of interest: N.E. Wijker reports grants from Australian National Health and Medical Research Council, during the conduct of the study. S. Vidmar reports grants from Australian National Health and Medical Research Council, during the conduct of the study. K. Grimwood reports grants from the Australian National Health and Medical Research Council, during the conduct of the study. P.D. Sly reports grants from Australian National Health and Medical Research Council, during the conduct of the study. C.A. Byrnes reports grants from Australian National Health and Medical Research Council and Faculty Research Development Fund, University of Auckland, New Zealand, during the conduct of the study. J.B. Carlin reports grants from Australian National Health and Medical Research Council, during the conduct of the study. P.J. Cooper reports grants from Australian National Health and Medical Research Council, during the conduct of the study. C.F. Robertson reports grants from Australian National Health and Medical Research Council, during the conduct of the study. R.J. Massie reports grants from Australian National Health and Medical Research Council, during the conduct of the study. M.P.C. Kemner van de Corput reports grants from Australian National Health and Medical Research Council, during the conduct of the study. J. Cheney reports grants from Australian National Health and Medical Research Council, during the conduct of the study. H.A.W.M. Tiddens reports grants from Australian National Health and Medical Research Council, during the conduct of the study; has provided lectures to Roche and Novartis; grants from CFF, Vertex, Chiesi and Vectura; fees for lectures and advisory board work from Gilead, outside the submitted work; in addition, has a patent PRAGMA-CF scoring system with royalties paid and 
is heading the Erasmus MC-Sophia Children's Hospital core laboratory lung analysis. FLUIDDA has developed computational fluid dynamic modelling based on chest CTs obtained from Erasmus MC-Sophia for which royalties are received by Sophia Research BV. C.E. Wainwright reports grants from Australian National Health and Medical Research Council, during the conduct of the study; and research grants from Vertex Pharmaceuticals Inc., Boehringer Ingelheim and Novo Nordisk Pharmaceuticals; fees for lectures and to attend meetings from Vertex Pharmaceuticals, DKBMed, Novartis Pharmaceuticals, University of Miami, Gilead Sciences Ltd and In Vivo Academy Limited; fees for consultancy, steering committee and advisory board work from Vertex Pharmaceuticals; fees for editorial board work from Thorax; fees for reviewing from BMJ and DKBMed; fees for transport and accommodation from Vertex Pharmaceuticals; and currently holds board positions on the International Advisory Board Vertex Pharmaceuticals P/L, and Associate Editor, Thorax and Respirology.

\section{References}

1 Armstrong DS, Grimwood K, Carlin JB, et al. Lower airway inflammation in infants and young children with cystic fibrosis. Am J Respir Crit Care Med 1997; 156: 1197-1204.

2 Sly PD, Brennan S, Gangell C, et al. Lung disease at diagnosis in infants with cystic fibrosis detected by newborn screening. Am J Respir Crit Care Med 2009; 180: 146-152.

3 Stick SM, Brennan S, Murray C, et al. Bronchiectasis in infants and preschool children diagnosed with cystic fibrosis after newborn screening. J Pediatr 2009; 155: 623-628.

4 Sly PD, Gangell CL, Chen L, et al. Risk factors for bronchiectasis in children with cystic fibrosis. $N$ Engl J Med 2013; 368: 1963-1970.

5 Loeve $\mathrm{M}$, Lequin $\mathrm{MH}$, de Bruijne $\mathrm{M}$, et al. Cystic fibrosis: are volumetric ultra-low-dose expiratory CT scans sufficient for monitoring related lung disease? Radiology 2009; 253: 223-229.

6 Loeve M, Hop WC, de Bruijne M, et al. Computed Tomography Cystic Fibrosis Survival Study G. Chest computed tomography scores are predictive of survival in patients with cystic fibrosis awaiting lung transplantation. Am J Respir Crit Care Med 2012; 185: 1096-1103.

7 Szczesniak RD, Li D, Su W, et al. Phenotypes of rapid cystic fibrosis lung disease progression during adolescence and young adulthood. Am J Respir Crit Care Med 2017; 196: 471-478.

8 Thia LP, Calder A, Stocks J, et al. Is chest CT useful in newborn screened infants with cystic fibrosis at 1 year of age? Thorax 2014; 69: 320-327.

9 Vandenbranden SL, McMullen A, Schechter MS, et al. Lung function decline from adolescence to young adulthood in cystic fibrosis. Pediatr Pulmonol 2012; 47: 135-143.

10 Wainwright CE, Vidmar S, Armstrong DS, et al. Effect of bronchoalveolar lavage-directed therapy on Pseudomonas aeruginosa infection and structural lung injury in children with cystic fibrosis: a randomized trial. JAMA 2011; 306: 163-171.

11 Byrnes CA, Vidmar S, Cheney JL, et al. Prospective evaluation of respiratory exacerbations in children with cystic fibrosis from newborn screening to 5 years of age. Thorax 2013; 68: 643-651.

12 Rosenow T, Oudraad MC, Murray CP, et al. A quantitative structural lung disease computed tomography outcome in young children with cystic fibrosis. Am J Respir Crit Care Med 2015; 191: 1158-1165.

13 Quanjer PH, Stanojevic S, Cole TJ, et al. Multi-ethnic reference values for spirometry for the 3-95-yr age range: the global lung function 2012 equations. Eur Respir J 2012; 40: 1324-1343.

14 Rosenow T, Mok LC, Turkovic L, et al. The cumulative effect of inflammation and infection on structural lung disease in early cystic fibrosis. Eur Respir J 2019; 54: 1801771.

15 Lima JA, Fischer GB. Foreign body aspiration in children. Paediatr Respir Rev 2002; 3: 303-307.

16 Maiz L, Nieto R, Canton R, et al. Fungi in bronchiectasis: a concise review. Int J Mol Sci 2018; 19: E142.

17 Silva JR, Jones JA, Cole PJ, et al. The immunological component of the cellular inflammatory infiltrate in bronchiectasis. Thorax 1989; 44: 668-673.

18 Cheng KK. The experimental production of bronchiectasis in rats. J Pathol Bacteriol 1954; 67: 89-98.

19 Goss CH, Sykes J, Stanojevic S, et al. Comparison of nutrition and lung function outcomes in patients with cystic fibrosis living in Canada and the United States. Am J Respir Crit Care Med 2018; 197: 768-775.

20 Ashkenazi M, Nathan N, Sarouk I, et al. Nutritional status in childhood as a prognostic factor in patients with cystic fibrosis. Lung 2019; 197: 371-376.

21 Corriveau S, Sykes J, Stephenson AL. Cystic fibrosis survival: the changing epidemiology. Curr Opin Pulm Med 2018; 24: 574-578.

22 Kuo W, Kemner-van de Corput MP, Perez-Rovira A, et al. Multicentre chest computed tomography standardisation in children and adolescents with cystic fibrosis: the way forward. Eur Respir J 2016; 47: 1706-1717.

23 Szczesniak R, Turkovic L, Andrinopoulou ER, et al. Chest imaging in cystic fibrosis studies: What counts, and can be counted? J Cyst Fibros 2017; 16: 175-185.

24 Kuo W, Soffers T, Andrinopoulou ER, et al. Quantitative assessment of airway dimensions in young children with cystic fibrosis lung disease using chest computed tomography. Pediatr Pulmonol 2017; 52: 1414-1423.

25 Perez-Rovira A, Kuo W, Petersen J, et al. Automatic airway-artery analysis on lung CT to quantify airway wall thickening and bronchiectasis. Med Phys 2016; 43: 5736. 